\title{
Effect of Lithium doping on the optical properties of monolayer $\mathrm{MoS}_{2}$
}

\author{
Nihit Saigal ${ }^{1, a}$, Isabelle Wielert ${ }^{1}$, Davor Čapeta ${ }^{2}$, Nataša Vujičić ${ }^{2}$, Boris V. Senkovskiy \\ 1, Martin Hell ${ }^{1}$, Marko Kralj ${ }^{2}$, and Alexander Grüneis ${ }^{1}$ \\ ${ }^{1}$ II. Physikalisches Institut, Universität zu Köln, Zülpicher Strasse 77, 50937 Köln, \\ Germany \\ 2 Center for Excellence for Advanced Materials and Sensing Devices, \\ Institute of Physics, Bijenička 46, 10000 Zagreb, \\ Croatia
}

The effect of Lithium atoms evaporation on the surface of monolayer $\mathrm{MoS}_{2}$ grown on $\mathrm{SiO}_{2} / \mathrm{Si}$ substrate is studied using ultra high vacuum (UHV $\sim 10^{-11}$ mbar) Raman and circularly polarized photoluminescence spectroscopies, at low Lithium coverage (up to $\sim 0.17$ monolayer). With increasing Li doping, the dominant $\mathrm{E}_{2 g}^{1}$ and $\mathrm{A}_{1 g}$ Raman modes of $\mathrm{MoS}_{2}$ shift in energy and broaden. Additionally, non zone-center phonon modes become Raman active. This regards in particular to double resonance Raman scattering processes, involving longitudinal acoustic (LA) phonon modes at the $M$ and $K$ points of the Brillouin zone of $\mathrm{MoS}_{2}$ and defects. It is also accompanied by significant decrease in the overall intensity and the degree of circular polarization of the photoluminescence spectrum. The observed changes in the optical spectra are understood as a result of electron doping by Lithium atoms and disorder-activated intervalley scattering of electrons and holes in the electronic band structure of monolayer $\mathrm{MoS}_{2}$.

a) Electronic mail : nihsai7795@gmail.com 
Monolayer (ML) Molybdenum Disulphide $\left(\mathrm{MoS}_{2}\right)$ is one of the most extensively studied members of the family of layered two dimensional (2D) materials. It shows several interesting physical phenomena such as, a direct bandgap leading to efficient photoluminescence $(\mathrm{PL})^{1,2}$, many body physical effects eg. observation of trions ${ }^{3}$ and biexcitons ${ }^{4}$, spin-valley coupling $^{5,6}$, exciton-phonon coupling ${ }^{7,8}$ etc. State-of-the-art devices like field effect transistors, solar cells, light emitting diodes etc. have been demonstrated based on these properties of $\mathrm{MoS}_{2} \cdot{ }^{9}$ Optical techniques like PL, Raman, reflectance and absorption spectroscopies have played a major role in $\mathrm{MoS}_{2}$ related research. ${ }^{10}$ However, the optical spectrum of this material is strongly affected by the presence of dopants and defects in the lattice. For instance, excitons bind to mono and bi- Sulfur vacancy defects in the $\mathrm{ML} \mathrm{MoS}_{2}$ lattice and lead to additional features in the PL spectrum apart from the nearly free exciton transition. ${ }^{11,12}$ Raman spectrum of $\mathrm{ML} \mathrm{MoS}$ is strongly affected by the presence of structural defects and additional phonon modes become Raman active in the disordered crystal. ${ }^{13}$ The peak energy and intensity of PL spectrum in $\mathrm{ML} \mathrm{MoS}_{2}$ can be tuned by chemical doping. ${ }^{14}$ Thus, it is important to systematically study the effect of dopants and disorder on the optical properties of ML $\mathrm{MoS}_{2}$.

Previously, several strategies have been used for doping $\mathrm{MoS}_{2}$ using different natured dopants, that include chemical treatment ${ }^{14-16}$, substitutional doping ${ }^{17,18}$, surface evaporation of dopants ${ }^{19,20}$ and gating in a transistor geometry ${ }^{3,21}$. It has been shown both theoretically and experimentally that surface adsorption of alkali metal atoms like $\mathrm{Li}, \mathrm{K}$, Cs etc. is an efficient way to reach very high levels of n-type doping $\left(\sim \mathbf{1 0}^{14} \mathbf{c m}^{-2}\right.$ electron density) in $\mathbf{2 D}$ materials. ${ }^{22,23}$ In bulk or in epitaxially grown layers (in epitaxial case even in ML limit) deposition of alkali metal atoms leads to ordered (intercalated) structures and possibility to precisely determine and correspondingly control charge transfer per added atom. ${ }^{24-26,28}$ On the other hand, for the adsorption of alkali metals or any other atoms or molecules on $\mathrm{ML} \mathrm{MoS}_{2}$ (or other 2D materials) transferred or directly grown on an amorphous $\mathrm{SiO}_{2}$ support, such quantification is far from straightforward, notably because intercalation or stable adsorption of well-defined ordered structure does not occur. ${ }^{18}$ Despite this difficulty, the investigation of atomic and molecular adsorption on single or few-layer $2 \mathrm{D}$ materials on a dielectric amorphous support such as $\mathrm{SiO}_{2}$, is of great importance with respect to possible 
applications, for example in sensors based on field effect transistors or in related devices for detection of changes in the optical response. ${ }^{19,29}$ Also, investigation of the optical spectra of doped ML $\mathrm{MoS}_{2}$ using circularly polarized light is important for design of valleytronic devices. ${ }^{5,6}$

Another interesting aspect of this material is the existence of phases which differ in their structural and electronic properties. In $2 \mathrm{H}$ phase of $\mathrm{MoS}_{2}$, Mo atoms have trigonal prismatic coordination and the crystal is semiconducting while in the $1 \mathrm{~T}$ phase, Mo atoms have octahedral coordination and the crystal is metallic. ${ }^{30,31} 1 \mathrm{~T} \mathrm{MoS}_{2}$ had been widely studied in bulk form due to its application as anode material in Lithium (Li) ion batteries. ${ }^{31}$ Recently, it has gained renewed interest due to its strong non-linear optical effects ${ }^{20}$, application in low resistance transparent contacts fabrication ${ }^{29}$, theoretical prediction of a small bandgap opening in the distorted $\left(1 \mathrm{~T}^{\prime}\right)$ form etc. ${ }^{32}$ Several strategies have been used to obtain the $1 \mathrm{~T}$ phase of $\mathrm{MoS}_{2}$ from its naturally occurring $2 \mathrm{H}$ phase, such as Li atom intercalation ${ }^{20,31}$, electron beam irradiation ${ }^{25}$ and electron transfer from a metallic substrate ${ }^{33}$ etc. Although, $\mathrm{Li}$ ion intercalation in $2 \mathrm{H}-\mathrm{MoS}_{2}$ has been extensively investigated for Li-ion batteries $^{26,31}$ and chemically/electrochemically exfoliated preparation of singlelayer $\mathrm{MoS}_{2}$ nanosheets ${ }^{15,29}$, the structural evolution of $\mathrm{MoS}_{2}$, especially the intermediate phases such as $1 \mathrm{~T}-\mathrm{MoS}_{2}$ and the occupancy of the $\mathrm{Li}$ atoms in it, is still not clear. ${ }^{27}$

In this letter, we discuss our investigation of the effect of in-situ evaporation of Li atoms on the surface of $\mathrm{ML} \mathrm{MoS}_{2}$ on $\mathrm{SiO}_{2} / \mathrm{Si}$ substrate under ultra high vacuum (UHV) (10 ${ }^{-11} \mathrm{mbar}$ ) conditions using micro-Raman and circularly polarized micro-PL spectroscopies at low temperatures $(\sim 6 \mathrm{~K})$. We observe that Li doping causes shifts and line-broadening of the dominant Raman modes of $\mathrm{MoS}_{2}$ and appearance of new Raman modes. Also, the intensity and circular polarization of PL spectrum of $\mathrm{MoS}_{2}$ are affected by Li doping. Our analysis of the Raman and PL spectra evolution with Li doping, indicates that the observed changes may arise from electron doping and disorder in the $\mathrm{ML} \mathrm{MoS}_{2}$ lattice caused by Li atoms. Here, we would like to comment that such a behavior has not been obtained in case of potassium doping in $\mathrm{ML} \mathrm{MoS}_{2}$.(see supplementary material)

Monolayer $2 \mathrm{H} \mathrm{MoS}_{2}$ films were grown in a homemade chemical vapor deposition (CVD) system from $\mathrm{MoO}_{3}$ and $\mathrm{S}$ precursors. Sulfur vapor was produced by heating 50-100 mg of Sulfur to $140{ }^{\circ} \mathrm{C}$ by a separate heater in front of the main furnace. Growth substrate, 


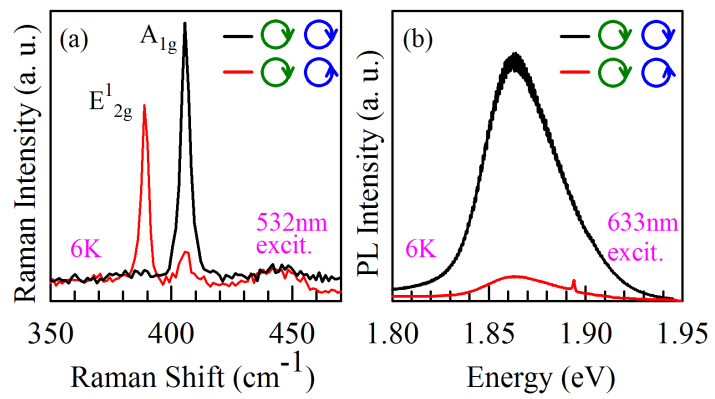

FIG. 1. Circular polarization resolved (a) Raman and (b) PL spectrum of ML $\mathrm{MoS}_{2}$. Green and blue circular arrows denote the excitation polarization of laser and detection polarization for the backscattered Raman and PL signals of the sample respectively.

$285 \mathrm{~nm} \mathrm{SiO}{ }_{2}$ on highly doped $\mathrm{Si}$, was placed in center of the furnace and heated to $750{ }^{\circ} \mathrm{C}$ during growth which typically lasted for 10 minutes. After growth, the substrate was cooled in furnace to $200^{\circ} \mathrm{C}$ in $\mathrm{Ar}$ stream before removal. The samples were mounted on sample holders and moved inside a home built UHV system ${ }^{35,36}$. They were initially annealed in the preparation chamber at $200-250{ }^{\circ} \mathrm{C}$ under UHV for $2-3$ hours to get rid of the surface residues and subsequently transferred to the analysis chamber equipped with a liquid He cryostat and an optical window for spectroscopic studies. The Li evaporation was done in steps inside the preparation chamber using high purity SAES Li getters with samples kept at room temperature. A quartz microbalance was used to estimate the thickness of deposited Li. $100 \mathrm{~s}$ of Li evaporation corresponds to $\sim 0.13 \mathrm{ML}$ coverage of Li. After each evaporation step the sample was put back in the analysis chamber and was given sufficient time to cool down before doing measurements. Samples were excited using either $532 \mathrm{~nm}$ or $633 \mathrm{~nm}$ laser beams. A combination of linear polarizers and quarter wave plate along with a commercial Renishaw spectrometer were used for circular polarization resolved Raman and PL spectroscopy (see supplementary material). The spectral and spatial resolution of the setup were $\sim 0.5 \mathrm{~cm}^{-1}$ and $\sim 5 \mu \mathrm{m}$ respectively.

Figures 1(a) and (b) show the results of circularly polarized Raman and PL measurements respectively. The green and blue circular arrows indicate the polarization of the excitation laser beam and that of the detected Raman or PL signal respectively. Clockwise rotation is for right circular polarization (RCP) and anti-clockwise for left circular polarization (LCP). Figure 1(b) shows the helicity dependence of the two main Raman modes. First note that 


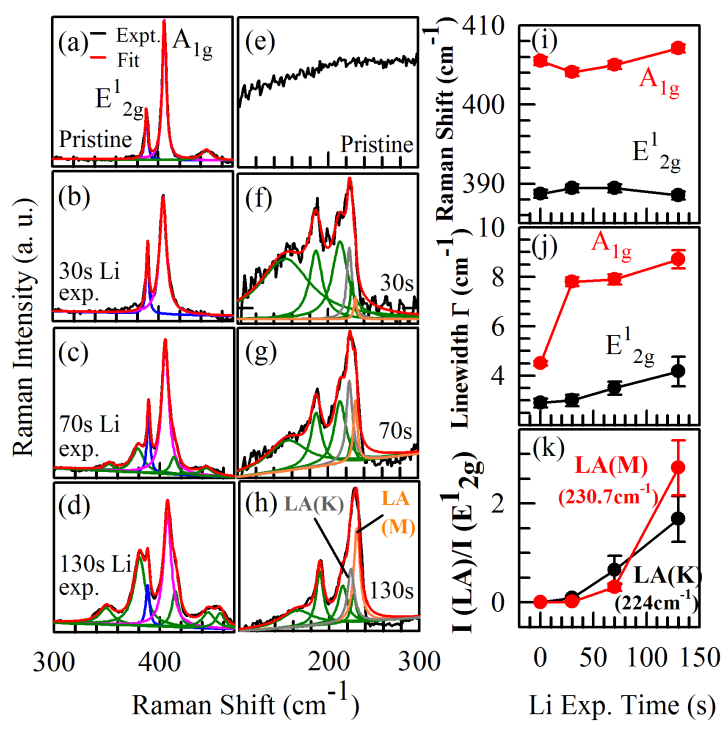

FIG. 2. (a)-(d) Experimental spectra (black) and Lorentzian fits (red) for the Raman peaks around the $\mathrm{E}_{2 g}^{1}$ (blue) and $\mathrm{A}_{1 g}$ (pink) Raman modes of $\mathrm{ML} \mathrm{MoS}_{2}$ for different Li exposure times. (e)-(h) Experimental Raman spectra and Lorentzian fits for the new Raman modes for different Li exposure times including Raman modes due to LA $(K)$ (dark grey) and LA( $M)$ (orange) phonons. Dark green peaks in panels (a) - (h) denote the individual Lorentzian peaks due to the other identified Raman modes (see supplementary material). (i) Peak energy and (j) linewidth of the $\mathrm{E}_{2 g}^{1}$ and $\mathrm{A}_{1 g}$ Raman modes as a function of Li exposure time. (k) Ratio of the intensity of Raman modes due to $\mathrm{LA}(K)$ and $\mathrm{LA}(M)$ phonons and that of the $\mathrm{E}_{2 g}^{1}$ mode, as a function of Li exposure time.

the energy difference between the two modes is $\sim 18 \mathrm{~cm}^{-1}$ which confirms the ML quality of our sample. ${ }^{37}$ We also observe that, while the $\mathrm{A}_{1 g}$ mode is dominant when the helicity of the detected Raman signal matches with that of the excitation laser, $\mathrm{E}_{2 g}^{1}$ mode is active only for the detection of opposite helicity. This helicity dependence comes from the symmetry properties of these phonon modes. ${ }^{38}$ These results are completely in line with previous studies ${ }^{38}$ which prove the feasibility of our setup and the good quality of our samples. The time reversal symmetry and absence of inversion symmetry together with a large spin-orbit (s-o) coupling in $\mathrm{ML} \mathrm{MoS}_{2}$ leads to optical spin-valley selection rules such that in the absence of inter-valley scattering (IVS) processes, a sample excited using a circularly polarized light with energy in resonance with an exciton transition, should show PL with the same helicity as the excitation source. ${ }^{5,6}$ Thus, a highly circularly polarized PL 
signal as shown in Fig. 1(b) is again an indication of the good quality of our pristine ML $\mathrm{MoS}_{2}$ samples.(supplementary material)

After certifying the good quality of our sample, we proceeded to stepwise evaporate Li atoms on the surface of $\mathrm{MoS}_{2}$, in-situ without exposure to air. After each step, the Raman and circularly polarized PL spectrum of the sample were obtained. Note that all measurements were done at low temperature (6K on the sample holder) to eliminate removal of Li atoms caused by laser heating. Low temperature is also relevant to make the Li atoms immobile, since at room temperature the charge transfer from $\mathrm{Li}$ atoms to $\mathrm{MoS}_{2}$ may vary due to mobility and/or loss of Li atoms. Figures 2(a) - (h) show the experimental spectra and Lorentzian fits to Raman spectrum for different Li exposure times. They show that on Li exposure, apart from $\mathrm{E}_{2 g}^{1}$ and $\mathrm{A}_{1 g}$ Raman modes, a number of new phonon modes become Raman active. These modes were previously observed in ion-bombarded $\mathrm{ML} \mathrm{MoS}_{2} \cdot{ }^{13}$ Based on previous studies, we can attribute majority of these modes to be due to phonons at the $M$ and $K$ points of Brillouin zone (BZ), which become Raman active in the presence of disorder in the $\mathrm{MoS}_{2}$ lattice (see supplementary material). ${ }^{13,39}$ Figures 2(i) and (j) respectively show the changes in peak energy and linewidth of the $\mathrm{E}_{2 g}^{1}$ and $\mathrm{A}_{1 g}$ Raman modes as a function of Li exposure time. The $\mathrm{A}_{1 g}$ mode shows an initial redshift in energy and an increase in linewidth after $30 \mathrm{~s}$ of $\mathrm{Li}$ exposure. In subsequent Li exposure steps, it blueshifts and keeps broadening. The $\mathrm{E}_{2 g}^{1}$ mode shows a small blueshift in the first Li exposure step and redshift in subsequent exposures. The $\mathrm{A}_{1 g}$ Raman mode in $\mathrm{MoS}_{2}$ is sensitive to the electron density and a redshift and broadening of this mode is an indication of electron doping of $\mathrm{ML} \mathrm{MoS}$ by Li atoms. ${ }^{21}$ We estimate the electron density in $\mathrm{MoS}_{2}$ at this stage to be $\sim 0.3 \times 10^{13} \mathrm{~cm}^{-2} \cdot{ }^{21}$ However, the subsequent blueshift of this mode with increasing Li exposure cannot be explained by electron doping. This may likely result form the breaking of translational symmetry of phonons due to disorder in the $\mathrm{MoS}_{2}$ lattice and their resultant spatial confinement. ${ }^{13,40}$ Some of the new Raman modes (Fig. 2(e) - (h)) can be identified as due to double resonance Raman scattering (DRR) processes involving the $\mathrm{LA}(M)$ and $\mathrm{LA}(K)$ phonons and defects in the $\mathrm{ML} \mathrm{MoS}_{2}$ lattice. ${ }^{13,39}$ The Raman modes due to $\mathrm{LA}(K)$ and $\mathrm{LA}(M)$ phonons have a dispersion of $-24.5 \mathrm{~cm}^{-1} / \mathrm{eV}$ and $-10.5 \mathrm{~cm}^{-1} / \mathrm{eV}$ respectively. ${ }^{39}$ We fixed the peak positions of these modes for $532 \mathrm{~nm}(2.33 \mathrm{eV})$ excitation and fitted our data using Lorentzian lineshape functions. In Fig. 2(k), we plot the ratio of the intensity of the Raman modes due to $\mathrm{LA}(K)$ and $\mathrm{LA}(M)$ phonons to that of the $\mathrm{E}_{2 g}^{1}$ Raman mode. This 

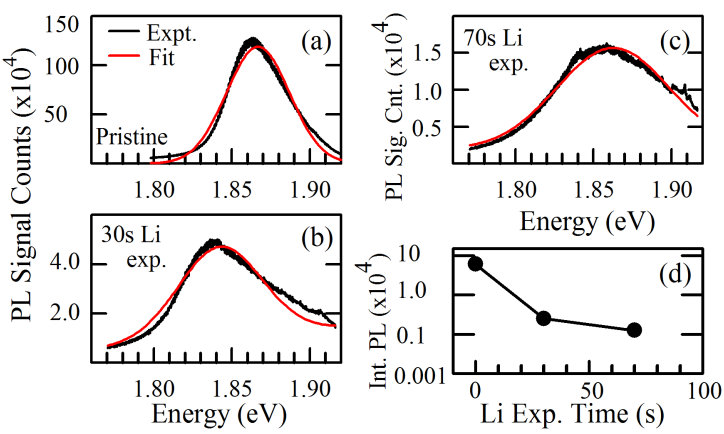

FIG. 3. (a)-(c) Experimental data (black) and Gaussian fits (red) for the circularly polarized $\mathrm{PL}$ spectrum (excitation and detection of right circular polarization) of $\mathrm{ML} \mathrm{MoS}_{2}$ for different $\mathrm{Li}$ exposure times. (d) The integrated PL intensity as a function of the Li exposure time.

ratio quantifies the disorder in the $\mathrm{MoS}_{2}$ lattice. ${ }^{13}$ An increase in this ratio with increasing $\mathrm{Li}$ exposure time shows that disorder in $\mathrm{MoS}_{2}$ lattice increases with increasing Li doping. As we mentioned above, Li doping can lead to the $2 \mathrm{H}$ to $1 \mathrm{~T}$ phase transition in $\mathrm{MoS}_{2}$ for high Li coverage $(\sim 2 \mathrm{ML}$ of $\mathrm{Li}) .{ }^{15,24}$ This transition is driven by the electron transfer from the $\mathrm{Li}$ atoms to $\mathrm{MoS}_{2}$, which results in stabilization of the $1 \mathrm{~T}$ phase relative to the $2 \mathrm{H}$ phase due to the absence of a bandgap in the former. ${ }^{24,30,31}$ Structurally, the transition involves a rearrangement of atoms in $\mathrm{MoS}_{2}$ which can cause lattice distortions and grain boundaries. ${ }^{25,31}$ Thus, the tendency to undergo the phase transition from $2 \mathrm{H}$ to $1 \mathrm{~T}$ form with Li doping could be responsible for the observed structural disorder in $\mathrm{ML} \mathrm{MoS}_{2} \cdot{ }^{15,24}$

Figures 3(a) - (c) show the experimental data and Gaussian fits for the circularly polarized PL of ML $\mathrm{MoS}_{2}$ for different Li exposure times. Note that we do not fit the data for the sample with 130 s of Li exposure because its PL has almost entirely vanished and the spectrum is dominated by background of the Raman signal. Also, the fits are not ideal because apart from the exciton transition, the PL may have contribution from an unresolved trion related transition. ${ }^{3,8,14}$ After the first $30 \mathrm{~s}$ of Li exposure, PL peak shows a redshift due to electron doping from Li atoms. ${ }^{3}$ After $70 \mathrm{~s}$ of Li exposure, it blueshifts. Such blueshift of the PL spectrum of ML $\mathrm{MoS}_{2}$ was also seen earlier in disordered samples. ${ }^{41}$ Figures $3(\mathrm{~d})$ shows the plot of integrated PL intensity with increasing Li exposure. We observe that the PL decays by more than three orders of magnitude after $70 \mathrm{~s}$ of Li exposure. The observed decay of PL intensity originates from different factors dominating at different stages of $\mathrm{Li}$ doping. Initially, electron doping due to charge transfer from Li atoms re- 
sults in trion formation (also evident from the redshift of the PL spectrum) and consequent decrease in the radiative recombination of excitons. ${ }^{3,14}$ With further increase in Li doping, structural disorder dominates as indicated by the changes in the Raman spectrum and also blueshift of PL spectrum. Structural defects in $\mathrm{MoS}_{2}$ can serve as sites for excitons to bind and decay non-radiatively. ${ }^{16}$ Also, disorder promotes IVS of electrons and holes to neighboring valleys from where they can possibly find paths for non-radiative decay. ${ }^{39}$ Finally, with further increase in Li doping, the change in the band structure around the K-point due to the $2 \mathrm{H}$ to $1 \mathrm{~T}$ phase transition leads to complete vanishing of the PL. ${ }^{15,34}$

Figure 4(a) shows the evolution of the circularly polarized PL with Li doping. As we discussed earlier, the circular polarization of the PL spectrum is due to spin-valley optical selection rules. The degree of circular polarization is defined as

$$
\rho=\frac{I(R C P)-I(L C P)}{I(R C P)+I(L C P)}
$$

where $\mathrm{I}(\mathrm{RCP}) / \mathrm{I}(\mathrm{LCP})$ denote the intensities of the RCP/LCP PL signals. ${ }^{6} \rho$ is a measure of valley polarization (VP) in $\mathrm{ML} \mathrm{MoS}_{2} .{ }^{6}$ Figure 4(b) shows a plot of $\rho$ around the PL peak energies, for different Li exposure times. It shows that $\rho$ decreases with increasing Li exposure. To make sure that we have not been probing a defective region of the sample by chance, we measured $30 \times 30 \mu \mathrm{m}$ spatial maps of $\rho$ in a typical region of the sample. Figures $4(\mathrm{c})$ and (d) show the maps for the pristine and $70 \mathrm{~s} \mathrm{Li} \mathrm{exposed} \mathrm{MoS}_{2}$ sample respectively. The maps show that for pristine sample $\rho$ is between $0.8-0.9$ while after $70 \mathrm{~s} \mathrm{Li} \mathrm{exposure,} \mathrm{it} \mathrm{decreases}$ to $0.5-0.6$.

The observed decay in the degree of circular polarization of PL (which is a measure of VP) can result from two main mechanisms. One is electron doping from $\mathrm{Li}$ atoms which may lead to increase in the electron-hole exchange interaction resulting in decrease in VP or valley depolarization. ${ }^{6}$ However, one does not observe a significant decrease in PL circular polarization for Potassium doping in ML $\mathrm{MoS}_{2}$.(see supplementary material) The second mechanism is IVS processes. IVS in ML $\mathrm{MoS}_{2}$ requires a simultaneous spin flip and a large change $(\sim \mathrm{K})$ in the crystal momentum of the excited carriers. ${ }^{6}$ Following resonant excitation of electron-hole pairs in $\mathrm{ML} \mathrm{MoS}_{2}$, the electrons can undergo a fast spin flip due to very small $(\sim 3 \mathrm{meV})$ s-o splitting of the conduction band. However, the s-o splitting of the valence band is large $(\sim 150 \mathrm{meV})$ and hence the hole spin flip is suppressed. ${ }^{6}$ Thus, pristine 


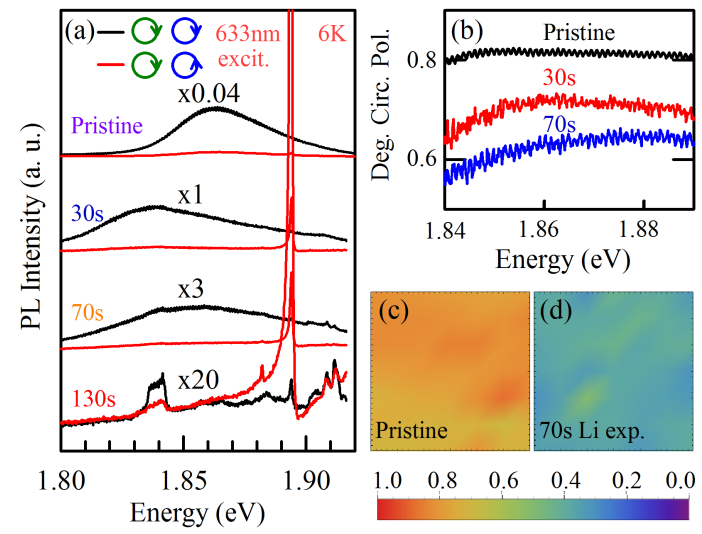

FIG. 4. (a) Evolution of the circularly polarized PL spectrum of $\mathrm{ML} \mathrm{MoS}_{2}$ for different Li exposure times. The spectra are vertically shifted and multiplied with the specified factors for clarity. (b) Degree of circular polarization of PL $(\rho)$ defined by eqn. 1, for different Li exposure times. $30 \times 30 \mu \mathrm{m}$ spatial maps of $\rho$ for (c) pristine and (d) $70 \mathrm{~s} \mathrm{Li} \mathrm{exposed} \mathrm{monolayer} \mathrm{MoS}_{2}$.

ML $\mathrm{MoS}_{2}$ shows a large VP. As we observed in the Raman spectra of Li doped $\mathrm{MoS}_{2}$, with increasing disorder, the DRR processes become active. These processes involve the scattering of an electron from $-K$ to $K$ valley and from $K(-K)$ to $Q$ valleys involving $\mathrm{LA}(K)$ and LA $(M)$ phonons respectively and defects. ${ }^{13,39}$ Scattering of electrons between $-K$ and $Q$ valleys may partly contribute to the decrease in PL intensity as we have already discussed. Valley depolarization on the other hand requires a simultaneous scattering of both electrons and holes between $-K$ and $K$ valleys. Even though hole scattering has to overcome a large spin-flip barrier, it can happen via the spin-degenerate $\Gamma$ valley. ${ }^{4,42}$ This would effectively lead to valley depolarization and hence the decrease in $\rho$.

In conclusion, we have observed that the evaporation of $\mathrm{Li}$ atoms on the surface of $\mathrm{ML} \mathrm{MoS}_{2}$ grown on $\mathrm{SiO}_{2} / \mathrm{Si}$ substrate causes significant changes in the optical spectrum of ML $\mathrm{MoS}_{2}$. With increasing Li doping, DRR processes become active leading to new peaks in the Raman spectrum. The circularly polarized PL spectrum shows a decrease in overall intensity and degree of circular polarization. These changes might be explained as a result of electron doping from Li atoms and IVS processes that become active because of disorder induced by $\mathrm{Li}$ atoms. Our results are a step towards understanding of the effect of doping and disorder on the optical spectrum of $\mathrm{ML} \mathrm{MoS}_{2}$ and similar 2D transition metal dichalcogenides. See supplementary material for a description of the optical setup and in-situ image of the sample, room temperature VP measurements, identified Raman modes in 
disordered $\mathrm{MoS}_{2}$, Raman and circularly polarized PL spectrum of Potassium doped ML $\mathrm{MoS}_{2}$ and circularly polarized Raman spectrum of Li doped ML MoS ${ }_{2}$.

Köln group acknowledges the ERC grant no. 648589 'SUPER-2D', DFG project CRC 1238 (project A1) and DFG project GR 3708/2-1. Zagreb group acknowledges financial support by the Center of Excellence for Advanced Materials and Sensing Devices and support of the Croatian Science Foundation (Grant No. IP-2016-06-3211).

\section{REFERENCES}

${ }^{1}$ A. Splendiani, L. Sun, Y. Zhang, T. Li, J. Kim, C. Y. Chim, G. Gali, and F. Wang, Nano Lett. 10, 1271 (2010).

${ }^{2}$ K. F. Mak, C. Lee, J. Hone, J. Shan, and T. F. Heinz, Phys. Rev. Lett. 105, 136805 (2010).

${ }^{3}$ K. F. Mak, K. He, C. Lee, G. H. Lee, J. Hone, T. F. Heinz, and J. Shan, Nat. Mater. 12, 207 (2012).

${ }^{4}$ C. Mai, A. Barrette, Y. Yu, Y. G. Semenov, K. W. Kim, L. Cao and K. Gundogdu, Nano Lett. 14, 202 (2013).

${ }^{5}$ T. Cao, G. Wang, W. Han, H. Ye, C. Zhu, J. Shi, Q. Niu, P. Tan, E. Wang, B. Liu, and J. Feng, Nat. Commun. 3, 887 (2012). (use et al.)

${ }^{6}$ K. F. Mak, K. He, J. Shan and T. F. Heinz, Nat. Nanotechnol. 7, 494 (2012).

${ }^{7}$ B. R. Carvalho, L. M. Malard, J. M. Alves, C. Fantini, and M. A. Pimenta, Phys. Rev. Lett. 114, 136403 (2015).

${ }^{8}$ N. Saigal and S. Ghosh, Appl. Phys. Lett. 107242103 (2015).

${ }^{9}$ Q. H. Wang, K. K. Zadeh, A. Kis, J. N. Coleman, and M. S. Strano, Nat. Nanotech. 14, 699 (2012).

${ }^{10}$ M. Ye, D. Winslow, D. Zhang, R. Pandey and Y. K. Yap, Photonics 2, 288 (2015).

${ }^{11}$ S. Tongay, J. Suh, C. Ataca, W. Fan, A. Luce, J. S. Kang, J. Liu, C. Ko, R. Raghunathanan, J. Zhou, F. Ogletree, J. Li, J. C. Grossman, and J. Wu, Sci. Rep. 3, 2657 (2013). (use et al.)

${ }^{12}$ N. Saigal and S. Ghosh, App. Phys. Lett. 109, 122105 (2016).

${ }^{13}$ S. Mignuzzi, A. J. Pollard, N. Bonini, B. Brennan, I. Gilmore, M. A. Pimenta, D. Richards and D. Roy, Phys. Rev. B 91, 195411 (2015). 
${ }^{14}$ S. Mouri, Y. Miyauchi and K. Matsuda, Nano Lett. 135944 (2013).

${ }^{15}$ G. Eda, H. Yamaguchi, D. Voiry, T. Fujita, M. Chen, and M. Chhowalla, Nano Lett. 11, 5111 (2011).

${ }^{16}$ M. Amani, D. H. Lien, D. Kiriya, J. Xiao, A. Azcatl, J. Noh, S. R. Madhvapathy, R. Addou, S. KC, M. Dubey, K. Cho, R. M. Wallace, S. C. Lee, J. H. He, J. W. Ager, X. Zhang, E. Yablonovitch, A. Javey, Science 350, 1065 (2015). (use et al.)

${ }^{17}$ J. Suh, T. E. Park, D. Y. Lin,D. Fu, J. Park,H. J. Jung, Y. Chen, C. Ko, C. Jang, Y. Sun,R. Sinclair,J. Chang,S. Tongay, and J. Wu, Nano Lett. 14, 6976 (2014). (use et al.)

${ }^{18}$ K. Zhang, S. Feng, J. Wang, A. Azcatl,N. Lu, R. Addou,N. Wang,C. Zhou, J. Lerach,V. Bojan, M. J. Kim, L. Q. Chen,R. M. Wallace, M. Terrones,J. Zhu,and J. A. Robinson, Nano Lett. 15, 6586 (2015). (use et al.)

${ }^{19}$ H. Fang,M. Tosun,G. Seol,T. C. Chang,K. Takei,J. Guo, and A. Javey, Nano Lett. 13, 1991 (2013).

${ }^{20}$ S. J. R. Tan, I. Abdelwahab, Z. Ding, X. Zhao, T. Yang, G. Z. J. Loke, H. Lin, I. Verzhbitskiy, S. M. Poh, H. Xu, C. T. Nai, W. Zhou, G. Eda, B. Jia and K. P. Loh J. Am. Chem. Soc. 13816632 (2017). (use et al.)

${ }^{21}$ B. Chakraborty, A. Bera, D. V. S. Muthu, S. Bhowmick, U. V. Waghmare and A. K. Sood, Phys. Rev. B 85 161403(R) (2012).

${ }^{22}$ C. A. Howard, M. P. M. Dean, and F. Withers, Phys. Rev. B 84, 241404(R) (2011).

${ }^{23}$ K. Dolui, I. Rungger, C. D. Pemmaraju, and S. Sanvito, Phys. Rev. B 88, 075420 (2013).

${ }^{24}$ D. N. Esfahani, O. Leenaerts, H. Sahin, B. Partoens and F. M. Peeters, J. Phy. Chem. C 119, 10602 (2014).

${ }^{25}$ Y. C. Lin, D. O. Dumcenco, Y. S. Huang and K. Suenaga, Nat. Nanotech. 9, 391 (2014).

${ }^{26}$ Y. Cheng, A. Nie, Q. Zhang, L. Y. Gan, R. S. Yassar, and U. Schwingenschlogl, ACS Nano 8, 11447 (2014).

${ }^{27}$ X. Wang, X. Shen, Z. Wang, R. Yu, and L. Chen ACS Nano 11, 11394 (2014).

${ }^{28}$ F. Ersan, G. Gökoğlu, and E. Aktürk, J. Phys. Chem. C 119, 28648 (2015).

${ }^{29}$ R. Kappera, D. Voiry, S. E. Yalcin, B. Brnach, G. Gupta, A. D. Mohite and M. Chhowalla, 
Nat. Mater. 13, 1128 (2014).

${ }^{30}$ J. A. Wilson and A. D. Yoffe, Advances in Phys. 18, 193 (1969).

${ }^{31}$ M. A. Py and R. R. Haering, Can. J. Phys 61, 76 (1983).

${ }^{32}$ X. Qian, J. Liu, L. Fu and J. Li, Science 3461344 (2014).

${ }^{33}$ X. Yin, Q. Wang, L. Cao, C. S. Tang, X. Luo, Y. Zheng, L. M. Wong, S. J. Wang, S. Y. Quek, W. Zhang, A. Rusydi and A. T. S. Wee, Nat. Commun. 8, 486 (2017). (use et al.)

${ }^{34}$ F. Xiong, H. Wang,X. Liu,J. Sun,M. Brongersma,E. Pop, and Y. Cui, Nano Lett. 156777 (2015).

${ }^{35}$ B. V. Senkovskiy, A. V. Fedorov, D. Haberer, M. Farjam, K. A. Simonov, A. B. Preobrajenski, N. Martensson, N. Atodiresei, V. Caciuc, S. Blügel, A. Rosch, N. I. Verbitskiy, M. Hell, D. V. Evtushinsky, R. German, T. Marangoni, P. H. M. van Loosdrecht, F. R. Fischer, and A. Grüneis, Adv. Electron. Mater. 31600490 (2017). (use et al.)

${ }^{36}$ A. Grüneis, B.V. Senkovskiy, A.V. Fedorov, M. Hell and S. Michel, Reference Module in Chemistry, Molecular Sciences and Chemical Engineering (Elsevier, 2017).

${ }^{37}$ C. Lee, H. Yan, L. E. Brus, T. F. Heinz, J. Hone, and S. Ryu, ACS Nano 4, 2695 (2010).

${ }^{38}$ S. Y. Chen, C. Zheng, M. S. Fuhrer and J. Yan, Nano Lett. 15, 2526 (2015).

${ }^{39}$ B. R. Carvalho, Y. Wang, S. Mignuzzi, D. Roy, M. Terrones, C. Fantini, V. H. Crespi, L. M. Malard and M. A. Pimenta, Nat. Commun. 8, 14670 (2017).

${ }^{40}$ H. Richter, Z. P. Wang and L. Ley, Solid State Commun. 39625 (1981).

${ }^{41}$ W. Shi, X. Zhang, X. L. Li, X. F. Qiao, J. B. Wu, J. Zhang and P. H. Tan, Chin. Phys. Lett. 33057801 (2016).

${ }^{42}$ G. Kioseoglou, A. T. Hanbicki, M. Currie, A. L. Friedman and B. T. Jonker, Sci. Rep. 6, 25041 (2016). 